\title{
Carlo Natali. II metodo e il trattato. Saggio sull'Etica Nicomachea. Roma: Edizioni di Storia e Letteratura, 2017
}

\author{
Manuel Berrón \\ Universidad Nacional del Litoral/Universidad Nacional de \\ Entre Rios, Argentina \\ mberron@fhuc.unl.edu.ar \\ iD http://orcid.org/0000-0001-6078-1994 \\ Matias Ezequiel Kogel \\ UniversidadNacionaldelLitoral,Argentina \\ kogel_matias_ezequiel@santafevirtual.edu.ar \\ iD http://orcid.org/0000-0002-5639-8586
}

Realizamos en primer lugar una presentación de la orientación general del libro en el marco de las investigaciones recientes sobre el tema de investigación. En ese sentido, mostramos sintéticamente el marco de las discusiones y el lugar de la obra en los desarrollos recientes. A continuación, expondremos la estructura general de la obra y luego, con algo de detalle, el contenido de los capítulos.

El libro del prestigioso aristotelista italiano Carlo Natali está dedicado al estudio de la Ética Nicomaquea (EN) bajo una renovada línea interpretativa de la filosofía práctica de Aristóteles. Natali reconoce que el punto de partida de su investigación se centró en el orden y la estructura del tratado pero que ello lo condujo a indagar en la metodología del mismo. Recordemos que la discusión sobre este tópico tiene un largo recorrido contemporáneo: la visión que preponderó desde 1960 sugiere que la metodología de la filosofía práctica es prioritariamente dialéctica, es decir, que argumenta sobre la base de opiniones reconocidas, que avanza resolviendo aporías y alcanza su validez por medio de la coherencia. Esta perspectiva, que sostiene la discrepancia entre la prédica metodológica de los Analíticos y la práctica científica de los tratados particulares, se fundó en los trabajos seminales de Burnet (1900), Le Blond (1939) y Owen (1961) y se desarrolló ampliamente con los de Cooper (1975), Barnes (1980), Nussbaum (1986) e Irwin (1988; 2009). Natali reconoce que él mismo sostuvo anteriormente esta tesis pero que, con el correr de las nuevas investigaciones en las décadas de 1980-90 dedicadas especialmente a las obras de biología (cf. Gotthelf y Lennox (1987) y Kullmann y Föllinger (1997)) fue detectando que Aristóteles estaría siguiendo las prescripciones metodológicas pautadas en los Analíticos en sus tratados particulares. En la década del 2000 la crítica hacia la omnipresencia de la dialéctica se acentuó y lo que reforzó este cambio de paradigma fue el importante libro de D. Charles (2000) sobre la relación entre esencia y explicación. En ese libro se encuentra en ciernes una nueva interpretación de la difícil relación entre definición y demostración estudiada en los capítulos 8-10 de Segundos Analiticos II. El debate sobre el tema es de suma actualidad, como lo atestigua la reciente publicación de D. Bronstein (2016) sobre el mismo tópico (naturalmente, Natali no contó con este material para el desarrollo de su trabajo). Pero la discusión es muy actual: además de los trabajos mencionados, los antecedentes para contextualizar el trabajo de Natali son los textos de Salmieri (2009), Karbowski (2013) y la compilación de D. Henry y K. M. Nielsen (2015), mientras que la discusión continúa con Angioni (2017), Falcon (2019) y Karbowski (2019) (éste último dedicado exclusivamente al método de la ética). Como se observa, Natali abreva de y discute con estas fuentes para aportar genuinamente a la vigente discusión sobre cuál es el método científico en la ética. Esta nueva línea de interpretación sugiere, 
como hemos dicho, que la metodología de la filosofía práctica respeta importantes rasgos de la metodología de los Analíticos, (y de ahí obtiene su nombre: "el método de los Analíticos"). El estudio de Natali se posiciona en este último momento de la discusión sobre la metodología de la filosofía práctica y busca ofrecer una renovada y más completa defensa de que la Ética Nicomaquea se organiza siguiendo atentamente el método "de los Analíticos" que propone el descubrimiento de las definiciones (cfr. APo. II 1-10). Si el seguimiento de dicha metodología no es manifiesto (y de hecho no lo es, lo que ha motivado tanta polémica), eso se debería, argumenta Natali, a que el texto tendría como destinatario un público amplio y no especializado. En síntesis, consideramos que la obra de Natali aporta valiosamente a la discusión contemporánea y se constituye como una referencia obligada para quienes quieran participar de la misma.

La obra cuenta con una introducción, siete capítulos, una conclusión, una lista de las abreviaturas utilizadas, una bibliografía, y dos índices: uno de nombres y otro de pasajes. En lo que concierne al contenido: el Cap. 1 versa sobre la estructura de $E N$, el Cap. 2 sobre el método utilizado y sobre la noción de felicidad, mientras que los restantes se ocupan primero de la noción de virtud en general (Cap. 3) y luego de las distintas virtudes: la justicia (Cap. 4), la prudencia (Cap. 5), el problema de la akrasía y el placer (Cap. 6) y sobre la amistad (Cap. 7).

El Cap. 1 está referido a la estructura de EN. Sin embargo, toda la primera sección se ocupa de mostrar la importancia de la $E N$-y también de la $E E$ - como las primeras obras de una disciplina independiente. En un interesante recorrido por el pensamiento moral precedente, a saber: las máximas de la poesía gnómica, Isócrates, Sócrates y Platón, Natali argumenta que en ninguna de estas formas de reflexión moral se encuentra la idea de sistema o reflexión sistemática sobre un tema particular. Estos conatos previos a la EN no llegan a constituir un campo independiente y autónomo. Contrariamente, la $E N$ logra esto: la independencia de un tipo de saber específico que se constituye como disciplina filosófica independiente distinta de la política y de la metafísica (p. 11-12). Natali retoma el tema del público de la $E N$ con más precisión para señalar que se trataría no de estudiantes de su grupo selecto (como quiere, v.gr., Salmieri, 2009, p. 318-319) sino de las élites políticas de su tiempo (lo mismo opina Karbowski, 2019, p. 136). Esto, sin embargo, no se observa en la $E E$. En lo que refiere a la estructura de $E N$, Natali señala que existe una línea clara en su argumento: primero la noción de bien humano y de allí a la definición de felicidad, luego a la virtud y a una discusión general de la virtud para, finalmente, una discusión pormenorizada de cada una de las virtudes (p. 18).

El Cap. 2 es particularmente relevante porque no sólo se aboca, tal como indica su título, a la teoría de la felicidad, sino porque tiene un apartado completo dedicado a exponer el "método de los Analíticos". En EN I Aristóteles investiga (zétesis) la naturaleza del bien supremo o felicidad, pero la investigación tiene por objetivo su definición. Y la definición de felicidad implica necesariamente la definición de virtud, con lo cual la búsqueda de la primera conlleva también la búsqueda de la segunda. Dado este objeto de investigación, Natali expone el método utilizado introduciendo un apartado (pp. 28-35) dedicado a su presentación. Allí retoma la ya mencionada tesis de Charles (2000) sobre $A P o$. II 1-2 y 8-13 que propone distintas etapas en la investigación y la búsqueda de las definiciones. En $A P o$. II 1 Aristóteles presenta los cuatro interrogantes de la investigación científica que, según él, luego se reducen a dos: de una parte, las preguntas por el hecho (hóti) y si es (ei ésti) y, de otra, las preguntas del porque (dióti) y de la esencia (tí éstin). Estas preguntas suponen un orden puesto que no se puede conocer la esencia de algo sin conocer antes su existencia (esto se hace explícito en $A P o$. II 8, 93a16-20). Para reforzar que en las éticas este método puede ser utilizado, Natali señala que tal procedimiento se encuentra explícitamente aludido en $E E$ II 1, 1220a15-22. Luego retoma $A P o$. II 8 y los ejemplos silogísticos referidos al eclipse y la distinción entre explicación del hecho y del porqué para mostrar que la búsqueda de la causa se relaciona con la búsqueda de la definición. A continuación, Natali refiere, aunque quizá demasiado sintéticamente, a la distinción entre definición nominal y definición real de $A P o$. II 10 para reforzar la conexión entre explicación y definición. Natali no hace un desarrollo mucho más amplio de estos tópicos y cierra su exposición diciendo que este método no es excluyente de otras opciones metodológicas como, por ejemplo, la inducción. A partir de la sección siguiente examina EN I para 
mostrar de qué manera se avanza desde la prueba de la existencia de un bien universal, i. e., la felicidad, por medio de un argumento ex scientiarum (p. 36) y se encamina así hacia el descubrimiento de la definición. Estos pasos comienzan con una definición nominal y examen de opiniones (EN I 4-5) para culminar con la conocida definición de felicidad de EN I 6. Por una parte, el silogismo del érgon no se apoya meramente en opiniones sino que parte de fenómenos empíricos (Natali los compara con los fenómenos celestes aludidos por Aristóteles en Cael. II 14, 297b23) y por otra, el silogismo introduce en la segunda premisa la causa formal; además, sostiene que la conclusión es la definición esencial del bien universal (pp. 45-7). Por esto, Natali argumenta que éste es un silogismo del hecho, donde la esencia es una conclusión y no tiene, por ahora al menos, una función explicativa. La definición, una vez hallada, debe poder funcionar de premisa y, así, resolver los problemas que sobrevengan al actuar como causa explicativa. Esto es lo que ocurre, según su interpretación, en los capítulos EN7-12. Por lo que toca al desarrollo anunciado en Cap. 1 sobre la estructura de $E N$, Natali confirma que hay un método certero que conduce de la definición de felicidad (EN I) a la de virtud (EN II) y que los libros III y IV se desarrollan en dependencia absoluta de estas definiciones. (p. 49-51).

En virtud del programa general previsto, el Cap. 3 se ocupa de estudiar la teoría de la virtud desarrollada, como se sabe, en EN II. En un primer apartado, se investiga las divisiones del alma y su correspondencia con las divisiones de la virtud. Siempre asegurando que el camino sea independiente de la metafísica y de la biología, la ética investiga dos partes del alma racional apoyándose en observaciones fácticas: el conflicto que existe entre una parte racional en sí misma y una parte que es racional pero desiderativa y, a la vez, capaz de obedecer a la anterior. Existe aquí una diferencia que da origen a dos tipos de virtudes: las intelectuales y las morales. Esta diferencia origina definiciones diversas que, sin embargo, se refieren exclusivamente a la parte racional del alma humana (p. 66). La distinción es fructífera porque desambigua la definición de felicidad al señalar que se tratará de una vida virtuosa, pero ahora con la especificidad de cada tipo de virtud, sea intelectual o moral. Luego hay una sección dedicada al modo en que se adquiere la virtud y continúa con otra dedicada a la definición de virtud. El capítulo termina con una sección dedicada al examen de la definición de la virtud en general y su relación con las virtudes particulares para mostrar de qué modo dicho examen vuelve la definición de felicidad menos abstracta y menos general. En este sentido, queda más claro qué sea el hombre virtuoso.

El Cap. 4 está dedicado al examen de la justicia. La primera y obvia observación es que el conjunto de libros $E N$ V-VII se corresponde con EE IV-VI. Natali nota que el estilo de este conjunto de libros es distinto del resto de $E N$. El libro $\mathrm{V}$ se divide en dos partes bien diferenciadas: en la primera se sigue el método de los Analíticos mientras que la segunda parte es una serie desordenada de observaciones en donde hasta puede observarse el método de resolución de aporías expuesto en EN VII 1. La segunda sección de este capítulo se dedica a estudiar el modo en que Aristóteles elabora la definición de la virtud de la justicia desde una definición nominal tomada de las opiniones reconocidas. En la sección siguiente se prueba la existencia de la justicia en general, lo que responde a la pregunta ei estin del método, y en la próxima la existencia de la justicia en particular. Esta investigación concluye con un reordenamiento de los elementos de la definición de virtud de EN II 6 (p. 108-9). El resto del capítulo está dedicado a señalar la multiplicidad de recursos utilizados y el desorden de la exposición. Hay un estudio de aporías, digresiones temáticas, más aporías y nuevas digresiones. Por estas razones Natali concluye que $E N \mathrm{~V}$ es un único libro, pero con multiplicidad metodológica.

El Cap. 5 está dedicado a la phrónesis, i. e., a EN VI, siempre en clave de su estructura y metodología. Natali repasa la discusión erudita sobre la estructura del libro para aseverar que Aristóteles en EN VI (i) completa la lista de las virtudes del alma y (ii) clarifica cuál es la capacidad intelectual que opera en conexión con lo irracional y ayuda a la virtud moral (p. 122). La conclusión del capítulo es que el libro se encuentra articulado con el recorrido iniciado en $E N$ I 13: explica las virtudes intelectuales y explica las virtudes morales por su relación con el órthos lógos. Además, Natali concluye que EN VI está en general bastante influenciado por el método de los Analíticos.

El Cap. 6 está dedicado al estudio de EN VII y X 1-5. Naturalmente, la lectura de este libro es relevante porque, como es sabido, en EN VII 1 se encuentra el célebre pasaje tomado por Barnes para construir 
su caracterización del método de los éndoxa. Su argumento es simple y convincente: reconoce el uso de los instrumentos de la dialéctica en dicho texto (p. 150) y el trato con aporías, pero su lectura es que el procedimiento empleado en el estudio de la akrasía (VII 1-11) se revela incapaz de alcanzar genuinas definiciones. En efecto, tal recorrido no alcanza a exponer los rasgos esenciales del objeto investigado y, por ello, es claramente insuficiente (p. 153). En lo relativo al estudio sobre el placer sostiene: (i) que respecto de $E N$ VII 12-15 no se alcanza una definición verdadera y propia del objeto (p. 159) y (ii) que sobre ENX 1-5 se encuentra un trato más cercano al de EN I y, por ello, más cercano al método de los Analíticos pero, sin embargo, no del todo concluyente en esta dirección.

El Cap. 7 es el menos extenso y se ocupa de los libros sobre la amistad, es decir, EN VIII y IX. En la primera sección del capítulo Natali discute su estructura y su conexión con el resto de la $E N$ : su conclusión es que el examen sobre la amistad tiene el aspecto de ser un tratado pensado para ser independiente, pero que no logra serlo y que, además, por estudiar una de las virtudes centrales en el desarrollo de la felicidad, no escapa al plan general de $E N$. En lo referido al método, Natali sostiene que el texto tiene un carácter expositivo y por momentos se recurre a una metodología que se asemeja a la dialéctica. Hay un examen de opiniones, una refutación de algunas y una búsqueda de las diferencias y las especies (EN VIII 2). Sin embargo, no se llega a definición alguna. El contexto es semejante al de $E N V$ donde no se llega a definir la justicia: aquí tampoco se llega a una definición verdadera de la amistad (p. 168-9). Finalmente, Natali se pregunta por la larga extensión de la reflexión aristotélica sobre la amistad y arroja la hipótesis de que se trata de una discusión heredada del Lisis de Platón y en la cual Aristóteles rivaliza y se distancia del maestro. Ciertamente, en la EN queda claro que las diferentes formas de amistad son el espacio natural en donde el hombre prudente desarrolla el plexo de virtudes abordadas en la obra.

En las conclusiones Natali rescata consecuencias tanto para la $E N$ como para el método científico. En primer lugar, que $E N$ está dirigida, en razón de su lenguaje y al contrario de $E E$, a un público no especializado. Además, el objetivo central de $E N$ sería de carácter protréptico y práctico sin abandonar su carácter filosófico. En este sentido, EN insta al hombre a buscar la virtud y obrar conforme a ella. Por lo que toca al estilo, el texto va de un estilo pulido y elocuente (EN I-IV) hacia uno que se degrada lentamente sin, no obstante, perder la unidad que comienza con el estudio de la felicidad, la virtud, luego las virtudes en general y luego las virtudes específicas $(E N \mathrm{~V}-\mathrm{X})$. Incluso los temas menos conectados como la debilidad del carácter, el placer y la amistad, están articulados por la necesidad de que sean conocidos por el hombre político (p. 179). Sin embargo, respecto de los libros sobre la amistad, Natali los considera un tratado autónomo destinado a polemizar contra la kallipolis de Platón: el contrapunto pasa por el hombre prudente de Aristóteles, pleno de virtudes sociales, por contraposición al guardián de Platón, un asceta en la isla de los Bienaventurados. Por otra parte, Natali confirma que Aristóteles a veces utiliza el método de los Analíticos y, a veces, el método de la resolución de aporías, como es el caso de EN VII. No obstante, el método dialéctico no es suficiente para alcanzar el objetivo propuesto en la $E N$, es decir, alcanzar definiciones, y para ello es preciso recurrir al método de los Analíticos. Natali documenta también la presencia de un uso "confirmativo" de la dialéctica que serviría para asegurar que las definiciones encontradas no resultan paradójicas (p. 182). Finalmente, sostiene acertadamente -y en contra de lo que se suele interpretar de modo erróneo- que, de acuerdo con Aristóteles, es posible un desarrollo estable del conocimiento en el plano de la ética al igual que en el plano de la física. Si bien la ética es una disciplina que persigue la acción, no tiene diferencia con ninguna ciencia en el nivel de la teoría. En efecto, las definiciones que en el plano teórico busca la ética no tienen una validez "para la mayoría" o "en ocasiones" sino siempre y absolutamente.

\section{REFERENCIAS}

Angioni, L. (2017). Explanation and method in Eudemian Ethics I.6. Archai, n ${ }^{\circ}$ 20, may- aug., 191-229. https:// doi.org/10.14195/1984-249X_20_8 
Barnes, J. (1980). Aristotle and the Methods of Ethics. Revue Internationale de Philosophie 133-134, 490-511.

Bronstein, D. (2016). Aristotle on knowledge and learning. Oxford: Oxford U. P.

Burnet, J. (1900). The Ethics of Aristotle, London: Methuen.

Charles, D. (2000). Aristotle on Meaning and Essence. Oxford: Oxford U. P.

Cooper, J. M. (1975). Reason and Human Good in Aristotle. Cambridge: Cambridge U. P.

Devereux, D. (2015). Scientific and ethical methods in Aristotle's Eudemian and Nicomachean Ethics. En Henry and K. M. Nielsen (Eds.), Bridging the Gap between Aristotle's Science and Ethics (pp. 130-47). Cambridge: Cambridge U. P.

Falcon, A. (2019). Aristotle's Method of Inquiry in Eudemian Ethics 1 and 2. En Bonazzi M., A. Ulacco y F. Forcignanò (Eds.), Thinking, Knowing, Acting: Epistemology and Ethics in Plato and Ancient Platonism (pp. 186-206). Boston: Brill.

Frede D. (2012). The endoxon mystique: What endoxa are and what they are not. Oxford Studies in Ancient Philosophy $43,85-215$.

Gotthelf, A. y J. G. Lennox (Eds.) (1987). Philosophical Issues in Aristotle's Biology. Cambridge: Cambridge U. P.

Henry, D. y Nielsen, K. (Eds.) (2015). Bridging the Gap Between Aristotle's Science and Ethics. Cambridge: Cambridge U.P.

Irwin, T. (1988). Aristotle's First Principles. Oxford: Clarendon Press.

Karbowski, J. (2013). Aristotle's Inquire into Natural Slavery.Journal of the History of Philosophy, Vol. 51 (3), 331-353. https://10.1353/hph.2013.0067

Karbowski, J. (2019). Aristotle's method in ethics: philosophy in practice. Cambridge: Cambridge U. P. https://10.10 $17 / 9781108303644$

Kullmann, W.y S. Föllinger (Eds.) (1997). Aristotelische Biologie, Intentionen, Methoden, Ergebnisse. Stuttgart: Verlag. Le Blond, J.M. (1939). Logique et méthode chez Aristote. Paris: Vrin.

Nussbaum, M. (1995). La fragilidad del bien: Fortun a y ética en la tragedia y la filosofia griega. Madrid: Visor.

Owen, G. E. L. (1980 [=1961]). Tithénai tà fainómena. En S. Mansion (Ed.), Aristote et les problemes de méthode (pp. 83-103). Louvain-la-neuve: Editions de L’Instut supérieur de philosophie.

Salmieri, G. (2009). Aristotle's Non- 'Dialectical' Methodology in the Nicomachean Ethics. Ancient Philosophy 29, 311-335. https://10.5840/ancientphil200929228 